\title{
Thibaut de Champagne et la tradition courtoise
}

\section{Danielle Quéruel}

\section{Citer ce document / Cite this document :}

Quéruel Danielle. Thibaut de Champagne et la tradition courtoise. In: Le Fablier. Revue des Amis de Jean de La Fontaine, $\mathrm{n}^{\circ} 27,2016$. pp. 27-30;

doi : https://doi.org/10.3406/lefab.2016.1276

https://www.persee.fr/doc/lefab_0996-6560_2016_num_27_1_1276

Fichier pdf généré le 09/03/2020 


\section{THIBAUT DE CHAMPAGNE ET LA TRADITION COURTOISE}

La poésie courtoise est l'une des innovations littéraires du Moyen Âge'. Chantant l'amour, ses souffrances et ses joies, selon des conventions exigeantes, cette poésie raffinée a fondé l'écriture et l'inspiration des poètes pendant les siècles suivants.

Thibaut IV, comte de Champagne, tient une place de premier plan dans cette production : auteur d'une œuvre poétique abondante, il développa au XIII ${ }^{\mathrm{e}}$ siècle dans ses II alais de Troyes et de Provins une cour raffinée ouverte cux arts et aux lettres. Heureux temps - comme le dit quelques années plus tard Jakemes auteur du Roman óu châtelain de Coucy et de la dame de Fayel - où les princes ne se contentaient pas d'être mécènes, mais étaient capables de chanter l'amour et de composer des vers ${ }^{2}$ ! Thibaut de Champagne fait partie de ces seigneurs lettrés qui furent à la fois princes et poètes ; il suit ainsi l'exemple de Guillaume IX d'Aquitaine, le premier troubadour reconnu, père d'Aliénor d'Aquitaine, il suit aussi celui de Richard Cœur de Lion, l'un des fils d'Aliénor d'Aquitaine, de Guillaume de Ferrières, vicomte de Chartres, de Guy, châtelain de Coucy... Le comte Thibaut de Champagne n'a pas laissé le souvenir d'un homme politique d'envergure et a souvent été critiqué pour ses hésitations à soutenir le royaume de France contre les Anglais ou pour le rôle minime qu'il joua pendant la croisade de 12391240 qui fut un échec. De son vivant, c'est en tant que poète qu'il fut reconnu : les Grandes Chroniques de France quelques années après sa disparition en 1253 font l'éloge de ses poésies " les plus delectables et melodieuses qui onques fussent ouïes en chanson et en vielle » et ajoutent qu' " il les fit copier sur les murs de sa grande salle à Provins et dans celle de Troyes et elles sont appelées les chansons du roy de Navarre ". Dante le cite comme l'un des plus éminents poètes de son époque, ce qui lui valut plus tard le surnom de Thibaut le Chansonnier.

Né en 1203, fils posthume du comte Thibaut de Champagne et de Blanche de Navarre, Thibaut fut élevé à la cour de France où il rencontra le poète Gace Brulé, chef de file des trouvères écrivant alors en langue d'oïl. Il y vécut auprès d'un prince cultivé, le futur roi Louis IX, et de la reine Blanche de Castille; une légende répandue par les Grandes Chroniques de France prétend que Thibaut en aurait été amoureux. Dans son comté de Champagne, il fit renaître l'éclat d'une cour qui dans la seconde partie du XII ${ }^{\mathrm{e}}$ siècle avait été brillante sous Henri le Libéral et Marie de Champagne et avait attiré intellectuels, écrivains et poètes venus de tous les pays. Assombrie quelques années par la mort soudaine du comte Thibaut III, survenue alors qu'il se préparait à diriger la quatrième croisade, la cour de Champagne retrouve son éclat avec son fils, Thibaut IV, qui a deux passions : les tournois et l'écriture poétique.

Il appartient à la deuxième génération de trouvères qui, en langue d'oïl, donnèrent naissance à une production poétique nouvelle. Du XII ${ }^{\mathrm{e}}$ au XIII ${ }^{\mathrm{e}}$ siècle les troubadours en langue occitane dans le Sud, puis les trouvères en langue d'oïl dans le Nord, ont pratiqué une poésie lyrique riche et complexe, alliant mots et mélodie, célébrant le plus souvent l'amour conçu comme un culte exigeant, comme un art d'aimer raffiné auquel on donnait le nom de « vraie amor » ou de "fin'amor", art d'aimer inaccessible au commun des mortels reposant sur une exaltation désespérée du désir, et que nous appelons aujourd'hui encore « amour courtois ». Thibaut de Champagne fut l'héritier de cette double tradition : d'abord celle des poètes occitans

1 Pendant les siècles médiévaux, la Champagne connut une effervescence culturelle et littéraire exceptionnelle. Riche en cuvres de toutes sortes, le patrimoine écrit qui est parvenu jusqu'à nous est immense : les noms de Chrétien de Troyes, inventeur du roman occidental, de Geoffroi de Villehardouin ou de Jean de Joinville qui donnèrent à la chronique son existence, de Bertrand de Bar-sur-Aube qui s'adonna à l'écriture de l'épopée, de Gace Brulé el de Thibaut de Champagne qui donnèrent à la tradition poétique courtoise ses plus belles pièces, de Rutebeuf, immense poète qui traduisit si bien les angoisses du $\mathrm{XIII}^{\mathrm{e}}$ siècle, puis plus tard d'Eustache Deschamps ou de Guillaume de Machaut, résonnent tous comme autant de preuves que cette terre de Champagne fut propice aux talents littéraires.

2 Jakemes, le Roman du châtelain de Coucy et de la dame de Favel, trad. A. Petit et F. Suard, La Ferté-Milon, Corps 9, « Textes littéraires médiévaux du Nord de la France ", 1986, p. 21. 
comme Jaufré Rudel, Bernard de Ventadour ou Bertrand de Born qui au XII ${ }^{\mathrm{e}}$ siècle fixèrent les règles formelles et l'inspiration thématique de la poésie courtoise, ensuite celle de la première génération de trouvères qui adaptèrent dans le Nord cette poésie et la firent entendre dans les cours de France et de Champagne. Gace Brulé en fut le chef de file, mais il faudrait nommer aussi Blondel de Nesles ou Huon d'Oisy et bien d'autres.

Thibaut de Champagne se détache parmi tous ces poètes par la qualité de son œuvre, le souci de la perfection formelle ainsi que par une écriture personnelle. Cette œuvre poétique est abondante - on lui attribue plus de 60 pièces - et diverse : comme ses prédécesseurs, il compose aussi bien des chansons de croisade et des lais religieux, des pastourelles, des débats mais ce sont les chansons d'amour - au nombre de 36 - qui constituent la majeure partie de son œuvre.

La chanson d'amour à cette époque procède d'une situation qui est toujours la même : le poète se dédouble en un amant qui aspire à l'amour d'une dame à qui il rend hommage, mais dont le silence, la froideur ou l'absence le font souffrir. L'amant-poète n'existe alors que par son amour et son besoin impérieux de l'exprimer grâce à ses vers. Les chansons composées déclinent dans des variations infinies tous les mouvements de son cœur et deviennent les médiatrices de son amour. Du XII siècle au milieu du XIII ${ }^{\mathrm{e}}$, les troubadours, puis les trouvères ont ainsi inventé et pratiqué une poésie lyrique qui montre comment le culte de la dame parée de toutes les vertus est le sujet unique qui guide l'inspiration du poète. Les chansons de Thibaut de Champagne se coulent tout naturellement dans ce moule ancien. L'agencement strophique et métrique des chansons en constitue le cadre : le plus souvent cinq strophes suivies d'un envoi, parfois ornées d'un refrain. Une mélodie accompagne les mots qui s'entrelacent dans une forme strophique élaborée, soutenus par des rimes, qui nous parlent d'amour. C'est en effet lui qui demeure le thème dominant du chant courtois et les conventions anciennes de la poésie courtoise persistent : l'amour s'impose à l'amant et le plonge dans un état de soumission envers sa dame, celle-ci est inaccessible et lointaine. L'amant tend vers la perfection, se soumet à toutes les volontés de la dame, mais n'obtient que rarement la récompense de ses efforts et de ses souffrances.

L'originalité de Thibaut de Champagne vient sans aucun doute en partie d'une prise de conscience que la chanson d'amour n'est pas un jeu, mais le moyen d'accéder à une plénitude de l'être. La dame aimée existeelle ? Elle garde tout son mystère. Jamais désignée, même par un surnom comme le faisaient les troubadours qui rivalisaient d'imagination pour la parer d'un « senhal », elle ne prend chair que par quelques traits rapides et génériques : elle est la « belle au beau corps », « la blonde aux fraîches couleurs ». Comparée à un " cerf aventureus " [chanson XVII] qui fait surgir le souvenir de la chasse au blanc cerf racontée par Chrétien de Troyes dans Érec et Énide, elle semble appartenir à un monde imaginaire. Paré de deux tresses blondes, blanc comme la neige, le cerf est enfermé dans un enclos dont l'entrée est périlleuse tout comme la dame est inaccessible à ceux qui l'aiment. L'écriture de Thibaut renouvelle ainsi les données courtoises.

La dame n'existe que par le désir du poète qui confie à sa chanson la mission de lui porter son message. Les premières strophes ainsi que les envois qui clôturent les chansons engagent avec cette dame un dialogue et en même temps nous montrent le rôle de la poésie. Écrire des vers devient pour le poète un besoin irrépressible pour avouer son amour et sa tristesse. Trop timide pour lui parler directement, il se sert des vers pour déclarer son amour et tenter de la convaincre de sa sincérité :

De nouveau il me faut chanter
Au moment où je suis le plus triste
Puisque je ne puis trouver grâce,
Je dois chanter à contre-cour.
Je n'ose parler à ma dame
Je fais de ma chanson un message
Pour elle qui est si courtoise et sage
Que je ne puis penser ailleurs ${ }^{3}$.
[chanson XII, str. $1^{4}$ ]

Dans la chanson III l'aveu est téméraire dans les premiers vers :
Si donc je dis follement
En ma chanson quelques-uns de mes désirs,
On ne doit pas m'en reprendre méchamment.
[chanson III, str. $1^{5}$ ]

Mais l'envoi relativise le pouvoir de l'amant en face de celui de la dame :

Chanson, dis-lui que tout cela est sans importance, Car, eût-elle cent fois juré ma mort, Je suis certain de rester en son pouvoir. [chanson III, envoi ${ }^{6}$ ]

Une certaine gravité pèse sur ces strophes qui sont emplies de larmes devant l'impossibilité d'être heureux :

Mes chants sont pleins de chagrin et de douleur A cause de vous, dame, que j'ai tant aimée. Je ne sais plus si je chante ou si je pleure... [chanson XVI, str. 3]

Je chante pour refouler mes larmes Et $\mathrm{j}$ 'attends la joie après la douleur. [chanson XXVI, str. 1]

Les citations sont tirces de la traduction d'Alexandre Micha, Thibaud de Champagne. Recueil de chansons, Paris, Klincksieck, 1991. Les textes originaux sont eux donnés en note d'après l'édition de Kathleen I. Brahney, The Lyrics of Thibaud de Champagne, New York/Londres, Garland Publishing, 1989.

$4 \quad$ Ibid., p. 72 : « De nouviau m'estuet chanter / El tens que plus sui marriz. / Quant ne pui merci trouver, / Bien doi chanter a enviz, / Ne je n'os a li parler ; / De ma chançon faz mesage, / Que tant est cortoise et sage / Que ne puis aillors penser. »

$5 \quad$ Ibid.,p. 64 : «Et pour ice, s'ai je dit folement / En ma chançon de ce que je voudroie, / Ne m'en doit on reprendre malement. »

6 Ibid.,p. 66: «Chançon, di li que tot ce n’a mestier, / Que, s’ele avoit cent foiz ma mort juree, / Si m'estuet il remaindre en son dangier. » 
La chanson se veut remède ou apaisement :

Je veux faire encore une chanson

Pour me consoler;

Pour celle qui me fait souffrir

Je veux renouveler mon chant,

J'ai besoin de chanter

Parce que, quand je ne chante pas,

Mes yeux souvent se mettent à pleurer.

[chanson XXXIII, str. $1^{7}$ ]

Désespoir ou stratégie amoureuse ? L'envoi est souvent conçu comme une supplique pour que la dame écoute ou chante ces vers qui parviendront jusqu'à elle, appel qui contient le fol espoir qu'ils feront naitre sa pitié ou son amour :

$\mathrm{Va}$, chanson, et ne t'attarde pas en route.

Demande à celle qui en a tout le pouvoir

D'être souvent chantée par elle.

[chanson XVI, envoi ${ }^{8}$ ]

Chanson, va et dis

A Blaison, mon ami

Qu'il te fasse chanter.

[chanson XVIII, envoi ${ }^{9}$ ]

Le procédé est subtil puisque, ici, c'est à un autre trouvère, Thibaut de Blaison, que Thibaut demande d'être son interprète.

Le poète délègue ainsi à sa poésie un rôle essentiel, faisant de sa chanson une médiatrice entre lui et sa dame, ce qui la rend réelle. La chanson est la meilleure stratégie pour conquérir le cœur de cette dame. Si elle apprécie ses vers, ne va-t-elle pas l'aimer ? Lui-même demeure incapable de faire cette démarche : soit il est « tout esbahis » en pensant à la beauté de celle qu'il aime et est incapable d'agir, soit il sombre dans le désespoir et aspire à la mort.

$\mathrm{Ce}$ sont ces mouvements où l'amour et la mort peuvent se confondre qui lui suggèrent ses plus beaux vers. La fin'amor en effet repose sur un paradoxe et aboutit à une impasse. Prônant un amour essentiellement stérile ou absent, elle est vouée à l'échec et à la mort :

\footnotetext{
Tant chante le rossignol

Qu'il tombe du haut de l'arbre.

Nul ne vit si belle mort

Si douce ou si agréable.

De même je meurs en chantant, à grands cris,

Puisque je ne puis être entendu de ma dame,

Et qu'elle ne daigne pas avoir pitié de moi.

[chanson $\mathrm{V}$, str. $\mathbf{1}^{10}$ ]
}

Reprenant la dualité symbolique que prête la tradition au rossignol depuis l'Antiquité, le poète évoque l'extase amoureuse qui va jusqu'à la mort et se compare à l'oiseau puisqu'il meurt lui aussi en exhalant son chant.

Dans la chanson XXXIV, c'est à nouveau dès le premier vers que le poète se compare à un animal fabuleux, la licorne. Conformément à la légende médiévale de la chasse à la licorne, l'amant se dit prisonnier, pris au piège d'amour et de la dame, donc voué à une mort certaine :
Je suis comme la licorne
En extase devant la jeune fille,
Dont elle ne détache pas ses regards.
Elle éprouve un si doux malaise
Qu'elle tombe sans connaissance en son giron.
Alors on la met à mort par traîtrise.
De même Amour et ma dame
M'ont blessé à mort, en vérité.
Ils ont mon cœur et je ne puis le reprendre.
[chanson XXXIV, str. $1^{11}$ ]

Dans la suite du poème, c'est comme un prisonnier que l'amant apparaît avec des séries sémantiques abondantes qui relèvent de l'allégorie à la manière du Roman de la Rose de Guillaume de Lorris écrit à la même époque. Les piliers de la douce prison sont faits de désir, les portes de beaux regards et les anneaux de bon espoir. Amour a la clef de la porte et les portiers sont Beau Semblant, beauté et Danger, c'est-à-dire Chasteté.

Thibaut de Champagne recourt à d'autres allusions souvent mythologiques ou romanesques, mais les dévie ou les transforme pour servir son propos. Il regrette ainsi que sa dame ne soit pas Thisbé puisqu'il souffre comme Pyrame ou détourne le personnage de Narcisse qui n'est plus que celui qui se noie : «Narcissus sui, qui noia tout par soi » écrit-il. Tous deux sont ici seulement représentatifs d'un amour voué au malheur. Il s'identifie aussi à Jason, à Tristan, à Roland ou à Olivier en cherchant le même effet : dire la mort qui l'attend. On peut ainsi résumer ces procédés en disant qu'à l'intérieur du carcan des conventions courtoises, Thibaut de Champagne apporte sa marque personnelle pensant avant tout à l'amour de l'écriture.

Cet exposé peut laisser croire que la poésie de Thibaut est grave et qu'il ne parle d'amour que de façon désespérée. On a parlé à son propos de badinage, de grivoiserie même ou de préciosité. Je voudrais dans les dernières lignes qui me restent présenter quelques pièces originales, celles que l'on appelle jeux-partis [9] ou débats [5]. Elles s'inscrivent dans un genre poétique dont la mode a fait fureur et qui était sans doute particulièrement bien représenté dans le Nord de la France. Fidèles aussi à la tradition courtoise,

7 Ibid., p. 56 : « Une chançon oncor vueil / Fere por moi conforter. / Pour cele dont je me dueil / Vueil mon chant renouveler. / Por ce ai talent de chanter / Car, quant je ne chant, mi œil / Tornent souvent a plorer. ")

$8 \quad$ Ibid., p. 8 : «Chançon, va t'en, garde ne remanoir. / Prie celi qui plus i a pooir / Que tu soies souvent par li chantee. "

9 Ibid., p. 36 : «Chançon va t'en tost et di / A Blazon, a mon ami, / Que il te face chanter. »

10 Ibid., p. 88 : « Li rosignox chante tant / Q il chiet mors de l’arbre jus. / Si bele mort ne vit nus, / Tant douce ne siplesant. / Autresi muir en chantant a hauz eriz, / Quant je ne puis de ma dame estre oïz, / N'ele de moi avoir pitié ne daigne. "

11 Ibid., p. 102 : «Aussi comme unicorne sui / Qui s'esbahist en regardant / Quant la pucele va mirant. / Tant est liee de son ennui, / Pasmee chiet en son giron ; / Lors l'ocit on en traîson. / Et moi ont mort d'autel semblant / Amors et ma dame, por voir : / Mon cuer ont, n'en puis point avoir. " 
elles parlent de l'amour et de ses exigences, mais cette fois sur un mode plaisant. La forme strophique de la chanson y est conservée, mais l'auteur procède ainsi : dans la première strophe est posée une question qui doit ensuite de strophe en strophe, d'argument en argument, trouver contradiction ou confirmation. Par exemple, deux amants aiment une même dame, l'un pour sa courtoisie, l'autre pour sa beauté. Lequel a le plus de chance de gagner son cœur [chanson XXXVII] ? Ou bien cet autre sujet est proposé : un amant a enfin obtenu le droit de se rendre près de sa dame. Que doit-il embrasser en premier lieu : la bouche ou les pieds de la dame ? Autre exemple : un amant aime une dame. Doit-il lui avouer son amour et risquer d'être repoussé ou bien se taire et souffrir en silence [chanson XLIV] ? Les situations sont souvent piquantes voire frivoles : si l'on obtient satisfaction de son amie, est-on plus amoureux avant ou après ? Ou enfin dans la pièce XLIII est présenté l'un des jeuxpartis les plus célèbres : vaut-il mieux souvent caresser son amie, la baiser, l'embrasser sans la voir la nuit sans allumer la chandelle ou lui parler et la voir tous les jours sans la caresser et sans la toucher ?

Ces controverses dénuées de profondeur psychologique relèvent d'une tradition double : le goût de la controverse et de la discussion dialectique qui caractérise le Moyen Âge et l'existence dès le $\mathrm{XII}^{\mathrm{c}}$ siècle d'un genre poétique occitan, le « partimen ", dans lequel le troubadour se livrait à des variations brillantes sur les contradictions et les exigences de l'amour courtois. Mais il nous paraît qu'il n'y a pas d'opposition réelle entre les chansons d'amour qui disent avec plus de gravité les difficultés d'aimer et ces débats qui font sourire des excès de l'amour courtois. Dans tous les cas, il s'agit pour le poète de répéter combien la puissance de l'amour est grande.

$\mathrm{Au} \mathrm{XIII}{ }^{e}$ siècle et sans doute grâce à des poètes comme Thibaut de Champagne, ces jeux-partis sont devenus des exercices de virtuosité et s'apparentent plutôt à des jeux de société mondains. Nous savons si peu de choses sur le fonctionnement des sociétés d'alors et sur les échanges qui se pratiquaient entre les cours qu'il serait imprudent d'aller plus loin dans cette analyse. Souvenons-nous cependant de l'existence d'un ouvrage composé à la fin du XII siècle, le Tractatus amoris attribué à un clerc André le Chapelain ${ }^{12}$. Il y met en scène avec un certain humour des rencontres qui pouvaient se passer à la cour de Champagne puisque les protagonistes en étaient entre autres dames la comtesse Marie de Champagne, sa mère Aliénor d'Aquitaine, sa belle-mère Alix de Champagne. L'auteur leur attribue une compétence extrême dans toutes les discussions portant sur l'amour. Par exemple, on écrit à la comtesse de Champagne qui passe ici pour une dame particulièrement experte en amour pour lui poser cette question : «Est-ce que le véritable amour peut exister entre époux et faut-il éprouver une grande jalousie entre amants? » [p. 111] Elle répond : " Nous disons et affirmons comme pleinement établi que l'amour ne peut étendre ses droits entre deux époux. Les amants en effet s'ac- cordent mutuellement toute chose gratuitement, sans qu'aucune obligation les pousse. » [p. 112]

C'est là la base de l'amour courtois chanté par les poètes et les romanciers. Il faut se souvenir que Marie de Champagne avait imposé quelques années auparavant à Chrétien de Troyes d'écrire un roman racontant l'amour adultère de Lancelot pour la reine Guenièvre, Lancelot ou le Chevalier de la charrette. Un peu plus loin dans ce traité un chapitre intitulé « De divers jugements d'amour » imagine que ces grandes dames sont consultées sur des points de casuistique amoureuse. On demande quels cadeaux une dame peut accepter de son amant sans manquer à la bienséance. Ou bien, si une dame est restée sans nouvelle de son amant parti à la croisade, doit-elle lui être fidèle ? La réponse est que, même s'il n'a pu lui écrire, elle doit toujours l'aimer car cet amant a agi avec prudence afin que leur secret ne soit pas dévoilé. Quant à la reine Aliénor qui s'est remariée avec le roi d'Angleterre âgé de vingt ans de moins qu'elle, on lui demande non sans ironie quel âge doit avoir l'amant idéal. L'ouvrage est évidemment dénué de tout fondement historique et a une visée essentiellement plaisante. Mais il a sans doute contribué à répandre l'idée que des cours d'amour existaient dans les châteaux médiévaux ce qui bien sûr ne peut être prouvé.

Une autre légende s'est attachée à Thibaut de Champagne, celle d'avoir fait revivre dans ses palais de Troyes et de Provins ces rencontres mondaines en attirant des poètes connus à cette époque. Des noms propres attestant que des liens existent entre lui et d'autres écrivains apparaissent dans ses poésies et tout particulièrement dans les jeux-partis. Thibaut s'y nomme parfois [début de XLII : " Roi Thibaut, seigneur, répondez-moi en chantant » ou XLIV «Bon roi Thibaut, seigneur, conseillez-moi » ou encore XLVII «Par Dieu, seigneur de Champagne et de Brie »]. Il construit alors ses pièces comme un dialogue avec un interlocuteur - parfois nommé lui aussi ou parfois seulement suggéré - et de strophe en strophe chacun défend une thèse qui concerne l'amour. On y trouve plusieurs fois Philippe de Nanteuil qui participa à la croisade de Thibaut en 1239, un certain Guy, clerc qui fut chancelier de Champagne en 1234, Jean de Brienne, roi de Jérusalem, poète lui aussi, Raoul de Soissons, vicomte de Coeuvres, qui participa également à la croisade. D'autres partenaires cités prouvent que ces poètes se connaissaient d'une région à l'autre : il met en scène Guillaume le Vinier, poète renommé d'Arras, Pierre Mauclerc, duc de Bretagne, surnommé Perron. Il y a là un ensemble de détails concrets, permettant d'imaginer des liens entre tous ces poètes, un jeu de société qui se faisait à la cour de Champagne peut-être à l'instigation de Thibaut lui-même.

Danielle Quíruel Université de Reims Champagne-Ardenne

12 André le Chapelain, Traité de l'amour courtois, trad. C. Buridant, Paris, Klincksieck, «Bibliothèque française et romane », 1974. 